\title{
ON THE LOCALISATION SEQUENCE IN $K$-THEORY
}

\author{
VICTOR SNATTH ${ }^{1}$
}

\begin{abstract}
A description of the boundary map in Quillen's localisation sequence is given in terms of classifying spaces of categories. Using this description the low dimensional part of the localisation sequence for a Dedekind domain is shown to coincide with the algebraically defined Bass-Tate sequence.
\end{abstract}

0. Introduction. Let $R$ be a ring with unit and $S \subset R$ a multiplicative set of central, nonzero divisors. Let $R_{S}$ be the localised ring. If $H$ is the exact category of finitely generated $R$-modules, $M$, having projective dimension $<1$ and $M_{S}=0$ there is a localisation sequence [G-Q] $(q>0)$

$$
\ldots \rightarrow K_{q+1}\left(R_{S}\right) \stackrel{\partial}{\rightarrow} K_{q}(H) \stackrel{a}{\rightarrow} K_{q}(R) \stackrel{b}{\rightarrow} K_{q}\left(R_{S}\right) \rightarrow \ldots
$$

The proof consists of utilising Quillen's Theorem B [Q, \&1] to realise $a$ and $b$ by means of a categorical quasi-fibration. This gives nice categorical descriptions of $a$ and $b$ but not of $\partial$. Using the techniques of [G-Q], [Q] it is shown (in \$1.5) how to realise $\partial$ and $a$ by means of a categorical quasi-fibration. In $\$ 2$ we apply this to compute $\partial$ when $R$ is a Dedekind domain with quotient field $R_{S}$.

1. Let $R$ be a ring, $S \subset R$ is a multiplicative set of central nonzero divisors and $H$ is the category of finitely generated $R$-modules, $M$, of projective dimension $<1$ such that $M_{S}=0$.

Let $G$ denote the following category. Its objects are surjections $L \rightarrow M \oplus B$ with $L, B \in \mathbf{P}(R)$, the category of finitely generated projective $R$-modules, and $M \in H$. An arrow $\left(L^{\prime} \rightarrow M^{\prime} \oplus B^{\prime}\right) \rightarrow(L \rightarrow M \oplus B)$ is represented by an equivalence class of diagrams

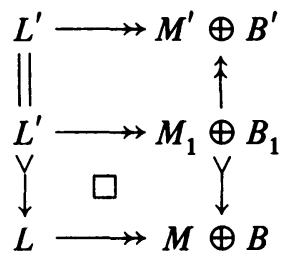

in which $\square$ denotes a pullback square and in which the vertical arrows are direct sums of arrows from $H$ and $P(R)$. Two such diagrams are equivalent if they are isomorphic by an isomorphism which is the identity on $M \oplus B$ and $M^{\prime} \oplus B^{\prime}$.

Received by the editors May 17, 1979 and, in revised form, July 9, 1979.

AMS (MOS) subject classifications (1970). Primary 18F25, 13F05; Secondary 55U40, 55N20.

Key words and phrases. Algebraic $K$-theory, category, Dedekind domain, localisation, tame symbol, valuation.

${ }^{1}$ Research partially supported by a grant from the Canadian N.R.C. 
Composition of two morphisms is accomplished by a pullback construction analogous to the composition in Quillen's $Q$-category [Q, \$2].

The category, $G$, was introduced in [G-Q, p. 230] and there the following is shown.

1.1. LemMA. The functor $h:(L \rightarrow M \oplus B) \mapsto M$ induces a homotopy equivalence $h: G \stackrel{\widetilde{\rightarrow}}{\rightarrow} Q H$.

Now consider the functor $g: G \rightarrow Q P R$ given by $g(L \rightarrow M \oplus B)=(B)$. This functor is fibred [G-Q]. That is, the functor $\Gamma: g^{-1}(B) \rightarrow B / g, \Gamma(L \rightarrow M \oplus B)=$ $\left(L \rightarrow M \oplus B, 1_{B}\right)$, has an adjoint, $\gamma$. Therefore $[Q, 81] \Gamma: g^{-1}(B) \rightarrow B / g$ is a homotopy equivalence.

Let us fix $B \in \mathbf{P}(R)$ and consider $g^{-1}(B)$. It has objects $(L \rightarrow M \oplus B)$, and a morphism $\left(L^{\prime} \rightarrow M^{\prime} \oplus B\right) \rightarrow(L \rightarrow M \oplus B)$ is an equivalence class of diagrams as in the category $G$ with the additional restriction that the endomorphisms of $B$ in the diagram should be the identity.

Define a second category $\tilde{g}^{-1}(B)$ whose objects are pairs $(L \rightarrow M \oplus B, B \rightarrow$ $L$ ) in which the first entry is an object of $G$, and the composition $B \longrightarrow L \rightarrow M \oplus$ $B$ is the inclusion of the second summand. Morphisms are equivalence classes of commutative diagrams of the following form.

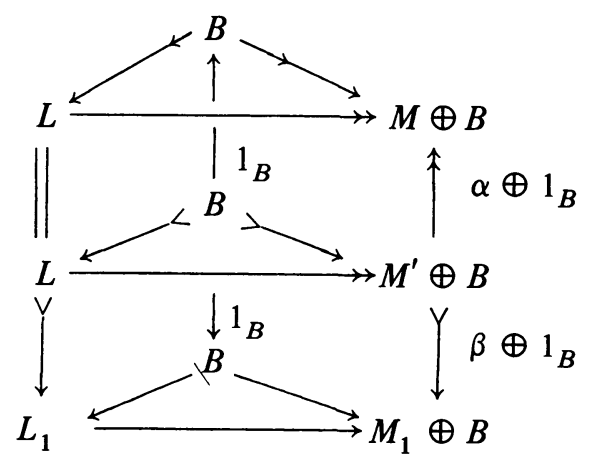

Composition of morphisms is defined as in $G$ and $g^{-1}(B)$.

1.2. Proposition. The functor $f: \tilde{g}^{-1}(B) \rightarrow g^{-1}(B)$ which forgets the morphism $B \longrightarrow L$ is a homotopy equivalence.

Proof. Fix an object $\left(L_{1} \rightarrow M_{1} \oplus B\right)$ in $g^{-1} B$ and choose a morphism $B \longrightarrow L_{1}$ so that $B \longrightarrow L_{1} \rightarrow M_{L} \oplus B$ is the inclusion of $B$. We will now show that $f /\left(L_{1} \rightarrow M_{1} \oplus B\right)$ is contractible. For an object of this category is represented by a diagram of the following form.

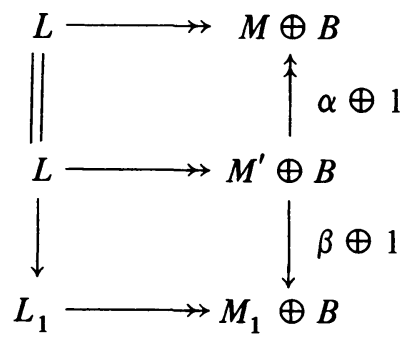


Hence the pullback square gives a natural morphism $B \rightarrow L$ from $B \longrightarrow L_{1}$ and $B \subset M^{\prime} \oplus B$. This gives a functor $a:\left(f / L_{1} \rightarrow M_{1} \oplus B\right) \rightarrow\left(1 / L_{1} \rightarrow M_{1} \oplus B\right)$ where 1: $g^{-1}(B) \rightarrow g^{-1}(B)$ is the identity functor. However forgetting $B \longrightarrow L$ gives a left inverse to $a$. Thus $B\left(f / L_{1} \rightarrow M_{1} \oplus B\right)$ must be contractible since it is a factor in $B\left(1 / L_{1} \rightarrow M_{1} \oplus B\right)$, and $1 / L_{1} \rightarrow M_{1} \oplus B$ has a final object.

Let $I=$ Iso $\mathbf{P}(R)$ the category of finitely generated projective $R$-modules and the isomorphisms. $I$ acts on $g^{-1}(0)$ by

$$
A+(L \stackrel{\pi}{\rightarrow} M)=(A \oplus L \stackrel{0+\pi}{\rightarrow} M)
$$

The category $\left\langle I, g^{-1}(0)\right\rangle$ has the same objects as $g^{-1}(0)$, and a morphism $(L \rightarrow M) \rightarrow\left(L^{\prime} \rightarrow M^{\prime}\right)$ is an isomorphism class of diagrams of the following form

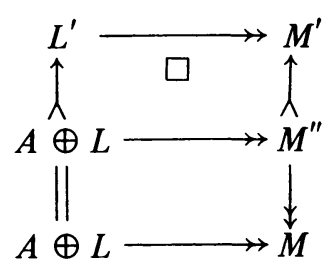

Here $A \in \mathbf{P}(R)$ and an isomorphism of diagrams of the above type is required on $A \oplus L$ to be the direct sum of an isomorphism on $A$ and one on $L$.

\subsection{Proposition. $\left\langle I, g^{-1}(0)\right\rangle$ is contractible.}

Proof. Direct sum makes $\left\langle I, g^{-1}(0)\right\rangle$ into an $H$-space, which is contractible by the proof of [G-Q, p. 227] as follows. The $H$-space is connected because $\left(L_{i} \rightarrow M_{i}\right)(i=1,2)$, maps to $\left(L_{1} \oplus L_{2} \rightarrow M_{1} \oplus M_{2}\right)$ by the inclusion of the $i$ th factor. Therefore we have only to show $\pi_{i}\left(B\left\langle I, g^{-1}(0)\right\rangle, 0\right)=0(i>1)$. However multiplication by two on these (abelian) homotopy groups is induced by the functor $F:(L \rightarrow M) \rightarrow(L \oplus L \rightarrow M \oplus M)$. The inclusion of the first summand gives a natural transformation from $\alpha: 1 \rightarrow F$ so that $B F \simeq 1$. Hence $2 x=x$ for $x \in \pi_{i}\left(B\left\langle I, g^{-1}(0)\right\rangle, 0\right)$ and the group must be zero.

1.4. THEOREM. The square

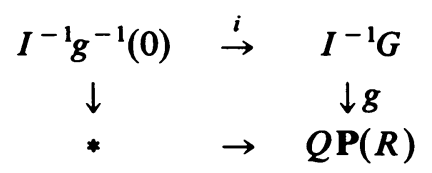

is homotopy cartesian, where $i(A, L \rightarrow M)=(A, L \rightarrow M)$.

Proof. By [Q, §1, Theorem 8] we must show that a morphism $0 \stackrel{\alpha}{\leftarrow} B^{\prime} \stackrel{\beta}{\rightarrow} B$ induces base change homotopy equivalences

$$
I^{-1} g^{-1}(0) \stackrel{I^{-1} \alpha^{*}}{\rightarrow} I^{-1} g^{-1}\left(B^{\prime}\right) \stackrel{I^{-1} \beta^{*}}{\rightarrow} I^{-1} g^{-1}(B)
$$

when $I^{-1} \alpha^{*}, I^{-1} \beta^{*}$ are induced by $\alpha^{*}, \beta^{*}$. 
By $\$ 1.2$ we may replace $g^{-1}(0)$ etc. by $\tilde{g}^{-1}(0)$. Also there are equivalences

$$
\tilde{g}^{-1}(0) \stackrel{j_{1}}{\rightarrow} \tilde{g}^{-1}(B) \stackrel{j_{2}}{\rightarrow} \tilde{g}^{-1}(0)
$$

given by $j_{1}(L \rightarrow M)=(L \oplus B \rightarrow M \oplus B)$ and

$$
j_{2}\left(\stackrel{j^{B}}{L^{\prime} \stackrel{\pi}{\longrightarrow} M^{\prime} \oplus B}\right)=\left(\text { coker } j \longrightarrow M^{\prime}\right) .
$$

With this identification $\beta^{*}: \tilde{g}^{-1}(B) \rightarrow \tilde{g}^{-1}\left(B^{\prime}\right)$ becomes the identity on $\tilde{g}^{-1}(0)$ because $\beta^{*}$ is pullback by $\beta$.

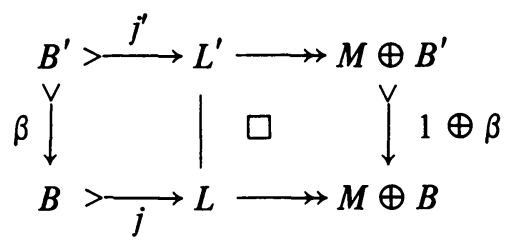

Furthermore $\alpha^{*}$ is given by sending $\left(L \rightarrow M \oplus B^{\prime}\right)$ to $\left(L \rightarrow M \oplus B^{\prime} \stackrel{\pi_{2}}{\rightarrow} M\right)$ which becomes the action of $B^{\prime}$ on $\tilde{g}^{-1}(0)$. However by Proposition 1.3 and [G-Q, p. 223] multiplication by $B^{\prime}$ on $I^{-1} \tilde{g}^{-1}(0) \simeq I^{-1} g^{-1}(0)$ is a homotopy equivalence.

1.5. CoRollary. There is a commutative diagram $(q>0)$

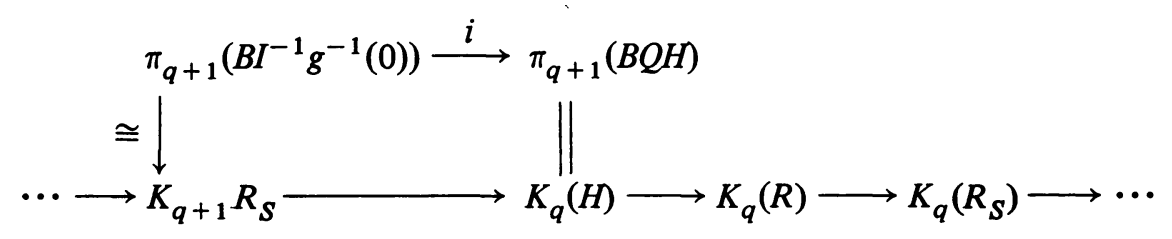

in which the row is the localisation sequence [G-Q, p. 233] and $i$ is induced by the functor $i(A, L \rightarrow M)=(M)$.

Proof. From the derivation of the localisation sequence [G-Q, pp. 229-233] we see that the equivalences $I^{-1} G \simeq I^{-1} Q H \simeq Q H$ transform the functor $g$ of $\$ 1.4$ into the map inducing $K_{*}(H) \rightarrow K_{*}(R)$. Hence Theorem 1.4 extends to the left the (quasi-) fibration sequence $B Q H \rightarrow B Q P(R) \rightarrow B Q P\left(R_{S}\right)$ and the result follows from the uniqueness, up to homotopy, of this extension.

1.6. Remark. Observe that Theorem 1.4 amounts to the assertion that $B I^{-1} g^{-1}(0)$ equals $\left(K_{0} R /\left(\operatorname{im}\left(K_{0} H\right)\right)\right) \times B G L R_{S}^{+}$.

2. Throughout this section set $R=A$, a Dedekind domain, with quotient field $F=R_{S}$. In this case devissage applied to $K_{*} H$ transforms the localisation sequence into the form

$$
\ldots \rightarrow K_{i} A \stackrel{b}{\rightarrow} K_{i} F \stackrel{\partial}{\rightarrow} \underset{P \triangleleft_{\max } A}{\bigoplus} K_{i-1}(A / P) \stackrel{a}{\rightarrow} K_{i-1} A \rightarrow \ldots
$$


Therefore we have $K_{1} F=F^{*}$ and $K_{0}(A / P)=Z(P)$, a copy of the integers. These identifications yield a homomorphism

$$
\text { ว: } F^{*} \rightarrow \underset{P \varangle_{\max } A}{\bigoplus} Z(P) \text {. }
$$

We will apply \$§1.4-1.5 to prove the following result (asserted by Quillen [Q, §7, Remark 5.17]). This result did not appear in [G-Q] as originally promised in [Q, ibid.].

2.2. TheOREM. The homomorphism of (2.1) is given by $\partial(x)=\Sigma \nu_{P}(x)$ where $x \in F^{*}$ and $\nu_{P}$ is the $P$-adic valuation.

Proof. Firstly set $J=$ Iso $P(F)$. Now let us give a functor $f: I^{-1} g^{-1}(0) \rightarrow J^{-1} J$ which is a homotopy equivalence on base-point components (both equal to $\left.B G L F^{+}\right)$. Having done this we will be able to use the description given in [G-Q, p. 224] of the loop in $\pi_{1}\left(B G L F^{+}\right)=F^{*}$ corresponding to $x \in A \subset F$ and thereby evaluate $\partial(x)$.

Assigning (Ker $\pi<L)$ to $(\pi: L \rightarrow M)$ gives an equivalence of categories with $I$-action between $g^{-1}(0)$ and the category, $\mathcal{L}$, of admissible layers in $\mathbf{P}(A)$. The functor $h\left(L_{0}<L_{1}\right)=L_{1} \otimes_{A} F$ is a homotopy equivalence of categories with $I$-action, $h: \mathcal{L} \rightarrow J$. This is because $\left(L \otimes_{A} F\right) / h$ equals the category of $A$-lattices which is called $X\left(L \otimes_{A} F\right)$ in [Q, $\$ 7$, Remark 5.17] and is shown there to be contractible. Hence $I^{-1} g^{-1}(0) \rightarrow I^{-1} J$ is a homotopy equivalence.

Now consider $I^{-1} J \rightarrow J^{-1} J$ induced by $I \rightarrow J$. This induces a homology isomorphism on base-point components of the classifying spaces, by [G-Q, p. 222]. However direct sum makes $B I^{-1} J$ and $B J^{-1} J$ into $H$-spaces (similarly base-point components) and so the map on base-point components is a homotopy equivalence.

Set $f$ equal to the composite $I^{-1} g^{-1}(0) \rightarrow I^{-1} J \rightarrow J^{-1} J$.

Now let $x \in A$. In $g^{-1}(0)$ we have two morphisms
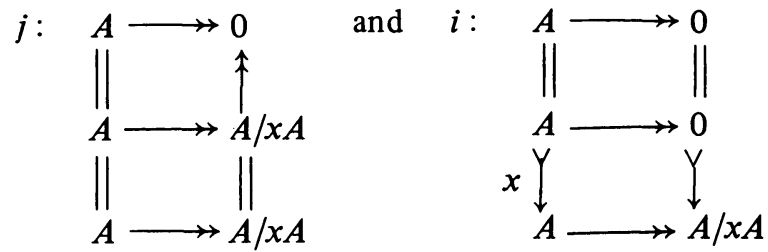

resulting in a loop $\left(1_{A}, i^{-1} \circ j\right) \in \pi_{1}\left(B I^{-1} g^{-1}(0) ;(A, A \rightarrow 0)\right)$. The functor $f$ takes this loop to the loop which is shown in [Q, \$2, Theorem 1] to determine $[A / x A] \in K_{0} H=\bigoplus_{P \triangleleft_{\max } A} K_{0}(A / P)$. By definition of the last (devissage) isomorphism [Q, §5, Corollary 1] $A / x A$ corresponds to $\Sigma \nu_{P}(x)$ as required.

2.3. Remark. The formula of $\$ 2.2$ agrees with that of [M, p. 123] for the algebraically defined coboundary in the localisation sequence of Bass and Tate.

Recall that the algebraically defined coboundary [M, p. 133]

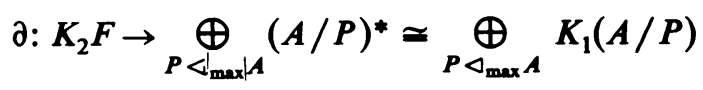


is given by the tame symbol. If $x, y \in F^{*}$ have Steinberg symbol $\{x, y\} \in K_{2} F$ the $P$-coordinate of $\partial\{x, y\}$ is given by $[\mathbf{M}, \S 11.5]$

$$
\partial\{x, y\}_{p}=(-1)^{v_{p}(x) v_{p}(y)} x^{v_{p}(y)} y^{-v_{p}(x)} .
$$

2.6. ThEOREM. The homomorphism of (2.4) is equal to the tame symbol whose $P$-coordinate is given by (2.5).

Proof. Recall that $K_{2} F$ is generated by Steinberg symbols $\{x, y\}\left(x, y \in F^{*}\right)$ and that $\{\}:, K_{1} F \otimes K_{1} F \rightarrow K_{2} F$ is equal to the topologically defined $K$-theory product given by Loday [L, \$2.2.3]. Furthermore the localisation sequence derived in [G-Q, p. 233] is a sequence of left $K_{*} A$-modules under Loday's product.

Also by naturality of the localisation sequence we can reduce to the local case. In this case let $\nu$ be the valuation associated to the maximal ideal. Let $x, y \in F^{*}$ satisfy $\nu(x)=n, \nu(y)=m$. Choose $z \in F^{*}$ so that $\nu(z)=1$. We may write $x=$ $z^{n} a, y=z^{m} b$ with $a, b \in A^{*}$.

We will write $K_{2} F$ additively and $K_{1}(A / P)$ multiplicatively.

Since $0=\{z, 1-z\}$ the bilinearity and skew-symmetry of the symbol yields $0=\left\{z, z^{-1}\right\}+\{z, 1-z\}+\{z,-1\}$. Therefore $\{z, z\}=\{z,-1\}$ and $\partial\{z, z\}=$ $\partial\{-1, z\}^{-1}=\left((-1)^{\nu(z)}\right)^{-1}=-1$, by the $K_{1} A$-module structure and Theorem 2.2.

Similarly we compute in general that

$$
\begin{aligned}
\partial\{x, y\} & =\partial(m n\{z, z\}+\{a, y\}-\{b, x\}) \\
& =(-1)^{m n} a^{\nu(y)} b^{-\nu(x)}
\end{aligned}
$$

which is the expression given in (2.5).

\section{REFERENCES}

[G-Q] D. Grayson (after D. Quillen), Higher algebraic K-theory. II, Lecture Notes in Math., vol. 551, Springer-Verlag, Berlin, 1976, pp. 217-240.

[L] J.-L. Loday, K-theorie algébrique et representations de groupes, Ann. Sci. Ecole Norm. Sup. (4) 9 (1976).

[M] J. W. Milnor, Introduction to algebraic K-theory, Ann. of Math. Studies, no. 72, Princeton Univ. Press, Princeton, N. J., 1971.

[Q] D. Quillen, Higher algebraic K-theory, I, Lecture Notes in Math., vol. 341, Springer-Verlag, Berlin, 1973, pp. 85-147.

Department of Mathematics, University of Western Ontario, London, Ontario N6A 5B9, CanAda 